\title{
Rosuvastatin Can Block Pro-Inflammatory Actions of Transgenic Human C-Reactive Protein Without Reducing its Circulating Levels
}

\author{
Jan Šilhavý, ${ }^{1}$ Václav Zídek, ${ }^{1}$ Vladimír Landa, ${ }^{1}$ Miroslava Šimáková, ${ }^{1}$ Petr Mlejnek, ${ }^{1}$ Vojtěch Škop, ${ }^{2}$ \\ Olena Oliyarnyk, ${ }^{2}$ Ludmila Kazdová, ${ }^{2}$ Massimiliano Mancini, ${ }^{3}$ Kathrin Saar, ${ }^{4}$ Herbert Schulz, ${ }^{4}$ \\ Norbert Hübner, ${ }^{4}$ Theodore W. Kurtz ${ }^{5} \&$ Michal Pravenec ${ }^{1}$ \\ 1 Institute of Physiology, Academy of Sciences of the Czech Republic, Prague, Czech Republic \\ 2 Center for Experimental Medicine, Institute for Clinical and Experimental Medicine, Prague, Czech Republic \\ 3 Department of Radiology, Oncology and Pathology, Sapienza Universita di Roma, Rome, Italy \\ 4 Max-Delbrück-Center for Molecular Medicine (MDC), Berlin, Germany \\ 5 University of California, San Francisco, CA, USA
}

\author{
Keywords \\ cardiac inflammation; C-reactive protein; \\ rosuvastatin; spontaneously hypertensive rat; \\ transgenic. \\ Correspondence \\ M. Pravenec, Institute of Physiology, \\ Academy of Sciences of the Czech Republic, \\ Vídeňská 1083, 14220 Prague 4, Czech \\ Republic. \\ Tel.: (420)241062297; \\ Fax: (420)241062488; \\ E-mail: pravenec@biomed.cas.cz
}

doi: 10.1111/1755-5922.12061

\begin{abstract}
SUMMARY
Aims: Statins have antiinflammatory effects and are known to decrease risk of cardiovascular events and to reduce serum levels of C-reactive protein (CRP), a widely studied biomarker and potential mediator of inflammation and heart disease. However, it is unclear whether statins can block pro-inflammatory effects of human CRP independent of their ability to reduce serum levels of human CRP. Here, we investigated whether rosuvastatin could block pro-inflammatory effects of human CRP without reducing circulating levels of human CRP. Methods and Results: We studied the antiinflammatory effects of rosuvastatin in spontaneously hypertensive rats (SHR) transgenically expressing human CRP (CRPtransgenic SHR) and in nontransgenic SHR lacking human CRP (nontransgenic SHR). The CRP-transgenic SHR is characterized by increased serum levels of human CRP and inflammation. In the CRP-transgenic strain, we found that rosuvastatin treatment decreased circulating levels of inflammatory response markers IL6 and TNF $\alpha$ without decreasing circulating levels of human CRP. In contrast, in the nontransgenic strain lacking human CRP, rosuvastatin treatment had little or no effect on IL6 and TNF $\alpha$ levels. Rosuvastatin also reduced cardiac inflammation and oxidative tissue damage, reduced epididymal fat mass, and improved adipose tissue lipolysis much more in the CRP-transgenic strain than in the nontransgenic strain. Conclusion: Rosuvastatin can protect against pro-inflammatory effects of human CRP in a manner that is not dependent on achieving a reduction in circulating levels of human CRP.
\end{abstract}

\section{Introduction}

C-reactive protein (CRP) is a well-known marker and potential mediator of inflammation that is associated with increased risk of cardiovascular and metabolic disease [1,2]. However, the extent to which CRP itself promotes inflammation and contributes to disease pathogenesis is highly controversial [2-8]. Although statins are known to reduce serum levels of CRP, it remains unclear whether any of the therapeutic benefits of statins are mediated by their ability to reduce serum concentrations of CRP. The results of "mendelian randomization" studies have suggested that the association between moderate increases in serum concentrations of CRP and increased risk of cardiovascular disease does not represent a cause and effect relationship [9,10]. However, mendelian randomization studies cannot address the potential therapeutic benefits of drugs that block pro-inflammatory actions of CRP in a manner that does not depend on reducing serum levels of CRP [11].

Because statins ordinarily reduce serum concentrations of CRP in humans, it is difficult to clinically investigate whether statins can block pro-inflammatory actions of human CRP independent of their effects on circulating levels of CRP. It is conceivable that statins may help protect against pro-inflammatory effects of human CRP in a manner that does not depend on reductions in serum levels of human CRP. The development of novel CRP inhibitors that can block inflammatory effects of human CRP in the absence of reductions in serum levels of CRP are of particular interest in this regard [12].

In the current study, in spontaneously hypertensive rats (SHR) harboring a transgene for human CRP, we investigated whether rosuvastatin could protect against inflammation dependent on the human CRP. We selected rosuvastatin, because results of the 
JUPITER trial suggested that treatment with rosuvastatin could reduce relative risk of cardiovascular disease in individuals with higher levels of CRP [13]. Because the human CRP is constitutively expressed by the transgene, statin treatment does not decrease circulating levels of human CRP in this model. Thus, this model can be used to test whether statins can block the ability of human CRP to promote inflammation independent of their effects on circulating levels of human CRP. Although mouse models are available that transgenically express human CRP, we chose to study the rat because of suggestions that the rat may be a more suitable model for investigating adverse biological actions of human CRP [14,15]. In addition to our primary goal of determining whether rosuvastatin could block pro-inflammatory actions of human CRP, we explored some secondary endpoints by determining whether rosuvastatin could protect against oxidative tissue damage and metabolic disturbances induced by human CRP. We also performed exploratory studies of the effects of rosuvastatin on gene expression levels in the liver to search for gene pathways that might be involved in the ability of rosuvastatin to protect against adverse effects of human CRP.

\section{Methods}

\section{Animals}

Transgenic SHR (hereafter referred to as CRP-transgenic SHR) were derived by microinjections of SHR ova with a previously described construct containing the cDNA for human CRP under control of the APOE promoter with the objective of driving expression of the CRP transgene in liver where CRP is normally produced $[16,17]$. We have chosen the SHR genetic background for expression of human CRP because the SHR is known as a model that is genetically predisposed to developing multiple features of the metabolic syndrome [16]. We measured circulating levels of human CRP and of the endogenous rat CRP using ELISA kits that distinguished between the two forms of CRP (Alpha Diagnostics International, San Antonio, TX, USA).

To investigate effects of rosuvastatin on inflammation caused by human CRP, we randomized 12-month-old CRP-transgenic SHR to groups with or without rosuvastatin treatment. We treated 12-month-old male CRP-transgenic SHR with rosuvastatin in the drinking water for 10 weeks. The concentration of rosuvastatin in the water was adjusted to deliver a daily rosuvastatin dose of approximately $5 \mathrm{mg} / \mathrm{kg} /$ day. A corresponding untreated control group of male CRP-transgenic SHR was given drinking water without rosuvastatin. In a similar fashion, we studied a group of nontransgenic SHR treated with rosuvastatin and an untreated group of nontransgenic SHR controls. In each group, we studied 5-8 animals. All rats were fed standard rat chow for the first 12 months and then switched to a high sucrose diet $(60 \%$ sucrose) to increase risk of developing metabolic disturbances during the 10 -week study period. The rats were housed in an air-conditioned animal facility and allowed free access to their chow and water. All experiments were performed in agreement with the Animal Protection Law of the Czech Republic and were approved by the Ethics Committee of the Institute of Physiology, Academy of Sciences of the Czech Republic, Prague.

\section{Primary Endpoint Methods General Markers of Inflammation}

Effects on serum levels of interleukin 6 (IL6) and tumor necrosis factor alpha $(\mathrm{TNF} \alpha)$ were used as coprimary endpoints for assessing the general ability of rosuvastatin to inhibit inflammation. Serum samples were obtained after 10 weeks of rosuvastatin therapy, and IL6 and TNF $\alpha$ were measured by rat ELISA kits (BioSource International, Inc., Camarillo, CA, USA).

\section{Secondary Exploratory Endpoints Cardiac Inflammation and Parameters of Oxidative stress}

To explore the ability of rosuvastatin to protect against cardiac inflammation and oxidative tissue damage, we measured the perivascular accumulation of monocytes and the tissue concentration of lipoperoxidation products in the heart. Lipoperoxidation products included conjugated dienes and thiobarbituric acid-reactive substances (TBARS) and were measured as previously described [18]. Perivascular accumulation of monocytes in the heart was determined by light microscopy observation. Rat hearts were cut along the long axis and processed for paraffin embedding. Multiple $4-\mu$ m-thick sections were cut and stained with hematoxylin and eosin. Perivascular monocytes were counted for each crosssectional arteriole found in the whole section, and the ratio between monocytes and each vessel was then derived.

\section{Parameters of Glucose and Lipid Metabolism}

To explore the ability of rosuvastatin to modulate adverse effects of human CRP on insulin, glucose, and lipid metabolism, we assessed glucose tolerance, insulin and lipid levels, and adipose tissue lipolysis and lipogenesis using methods similar to those previously reported [16].

\section{Gene Expression Profiling}

To explore for candidate gene pathways that might be involved in mediating the ability of rosuvastatin to protect against adverse effects of human CRP, we performed gene expression profiles in livers isolated from CRP-transgenic SHR and nontransgenic SHR treated with rosuvastatin or placebo. We studied hepatic tissue because the CRP transgene was expressed in the liver and rosuvastatin is most highly concentrated in liver relative to other tissues [19]. Detailed descriptions of the gene expression profiling methods and analysis are provided in Data S1.

\section{Statistical Analysis}

Our goal was to determine whether antiinflammatory effects of rosuvastatin treatment would be greater in the transgenic strain expressing human CRP than in the nontransgenic strain. Toward this end, we used two-way ANOVA to test for treatment $\times$ strain interactions, and if present, we determined whether rosuvastatin 
inhibited inflammation to a greater extent in the CRP-transgenic strain than in the nontransgenic strain. For variables showing evidence of treatment $\times$ strain interaction, we used Holm-Sidak testing that adjusts for multiple comparisons to determine whether the antiinflammatory effects of rosuvastatin were significant in the nontransgenic SHR strain and in the transgenic SHR strain expressing human CRP. For measurements of cardiac monocyte infiltration where only three experimental groups were studied, the results were analyzed by one-away ANOVA with adjustments for multiple comparisons by Holm-Sidak testing. Results are expressed as means \pm SEM. The analysis of gene expression levels was performed using a two-way ANOVA approach followed by a false discovery rate (FDR) multiple testing correction procedure (see Data S1 for details).

\section{Results}

\section{Effects of Rosuvastatin on Serum Levels of Human CRP and Rat CRP}

Figure 1 shows the serum levels of human CRP and endogenous rat CRP in the different experimental groups. There was no significant treatment $\times$ strain interaction effect and no significant rosuvastatin treatment effect on serum levels of human CRP. Because the human CRP transgene was under control of an APOE promoter, rosuvastatin treatment was not expected to have much, if any, effect on serum levels of human CRP. In contrast, a significant effect of rosuvastatin treatment was observed $(P=0.013)$ on serum levels of endogenous rat CRP in both strains.

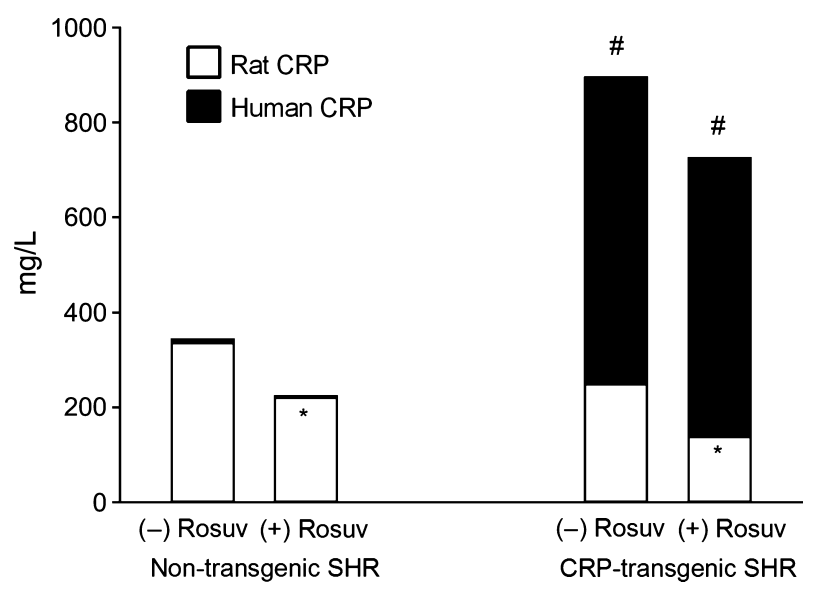

Figure 1 Serum levels of endogenous rat CRP (open bars) and human CRP (solid bars). As expected, human CRP was clearly detectable only in the CRP-transgenic strain. \#Significant effect of strain on human CRP levels in the two-way ANOVA $(P<0.0001)$. There was no significant strain effect on serum levels of rat CRP. *Significant rosuvastatin treatment effect on serum levels of rat CRP $(P=0.013)$. The rosuvastatin induced decreases in rat CRP were similar between the nontransgenic SHR strain and the CRP-transgenic rat strain. There was no significant treatment $\times$ strain interaction effect on serum levels of either rat CRP or human CRP. CRP, C-reactive protein; SHR, spontaneously hypertensive rat; and Rosuv, rosuvastatin.

\section{Effects of rosuvastatin on inflammation induced by human CRP}

Figure 2 (top panel) shows the effects of rosuvastatin treatment on serum levels of IL6 and TNF $\alpha$. Two-way ANOVA showed significant treatment $\times$ strain interaction effects on serum levels of both IL6 $(P=0.0015)$ and TNF $\alpha(P=0.048)$. In the transgenic SHR strain expressing human CRP, rosuvastatin induced significant decreases in serum levels of IL6 and TNF $\alpha$ whereas in the nontransgenic SHR strain, rosuvastatin treatment had little or no effect on IL6 and TNF $\alpha$.

\section{Effects of Rousvastatin on CRP-Induced Inflammation and Oxidative Tissue Damage in the Heart}

Cardiac inflammation was assessed by measuring perivascular monocyte infiltration in the heart (mean number of monocytes per cardiac arteriole). Figure 2 (bottom left panel) shows that transgenic expression of human CRP induced an increase in perivascular accumulation of monocytes in the cardiac tissue of untreated rats. In the untreated human CRP-transgenic strain, the number of perivascular monocytes in cardiac tissue was significantly greater than in the untreated nontransgenic strain $(P<0.05)$. In the CRP-transgenic rats treated with rosuvastatin, the number of perivascular monocytes in cardiac tissue was significantly lower than in untreated CRP-transgenic rats $(P<0.05)$ and was not different from that in untreated nontransgenic controls.

We also assessed the effects of rosuvastatin on human CRPinduced oxidative tissue damage in cardiac tissue by measuring lipoperoxidation products in the heart (Figure 2, bottom right panel). Two-way ANOVA showed a significant treatment $\times$ strain interaction effect on oxidative tissue damage in the heart as judged by rosuvastatin-induced changes in conjugated dienes $(P=0.0068)$. In the transgenic SHR strain expressing human $\mathrm{CRP}$, rosuvastatin induced a significant decrease in conjugated dienes in the heart whereas in the nontransgenic SHR strain, rosuvastatin treatment did not have an effect on conjugate dienes (Figure 2, bottom right panel). There was no significant effect of rosuvastatin on TBARS in the heart (data not shown).

\section{Metabolic Effects of Rosuvastatin in CRP- Transgenic SHR}

There was a significant treatment $\times$ strain interaction effect on body weight as rosuvastatin treatment tended to promote greater weight gain in the nontransgenic strain while it tended to attenuate weight gain in the CRP-transgenic strain (Table 1). However, the absolute effects of rosuvastatin on body weight within each strain were small and did not achieve statistical significance after adjustment for multiple comparisons. Significant treatment $\times$ strain interaction effects were observed on insulin levels $(P=0.021)$ and on the insulin levels measured during the glucose tolerance test $(P=0.007)$. Rosuvastatin treatment decreased insulin levels in the CRP-transgenic strain but had little or no effect on insulin levels in the nontransgenic strain. No significant treatment $\times$ strain interaction effects were observed on either glucose, 

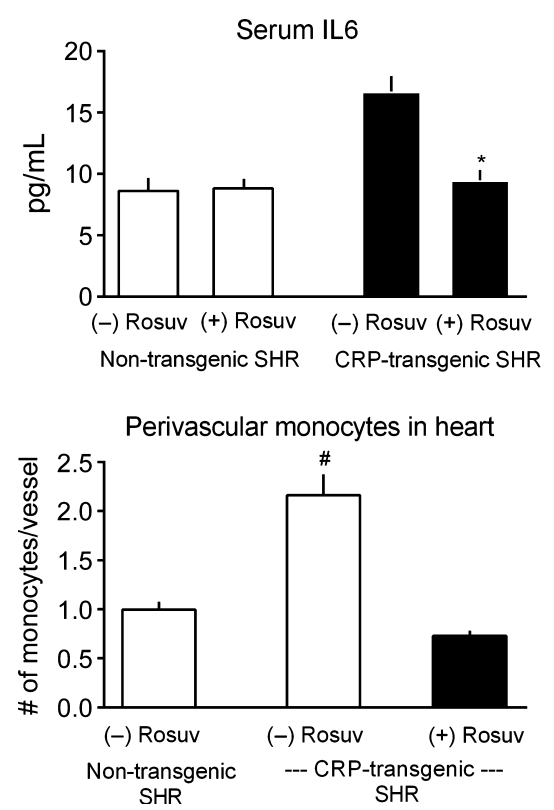

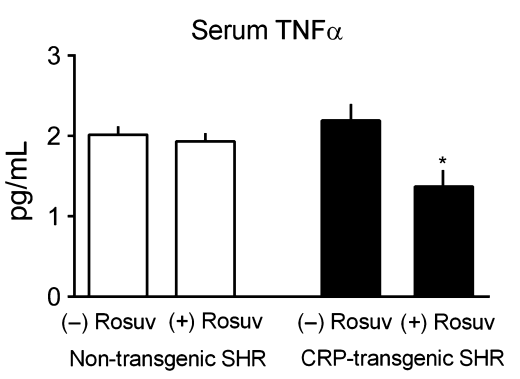

Conjugated dienes in heart

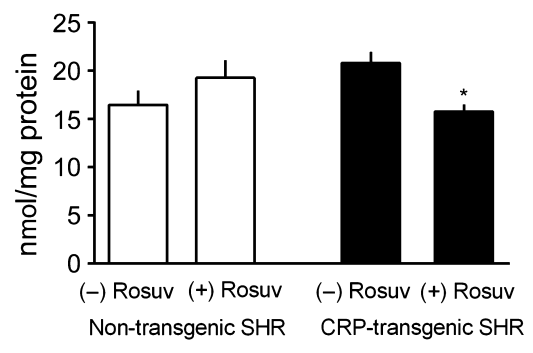

Figure 2 Top panel: serum levels of IL6 and TNF $\alpha$. Two-way ANOVA showed a significant treatment $\times$ strain interaction effect on IL6 levels $(P=0.0015)$ and on TNF $\alpha$ levels $(P=0.048)$. *Rosuvastatin treatment induced a significant reduction in IL6 and TNF $\alpha$ levels in the CRP-transgenic SHR strain. Rosuvastatin did not affect IL6 and TNF $\alpha$ levels in the nontransgenic SHR strain. Bottom panel: Cardiac inflammation and oxidative tissue damage. Cardiac inflammation was assessed by measuring perivascular monocyte infiltration (mean number of monocytes per vessel). One-way ANOVA showed significant group differences in the number of perivascular monocytes per heart vessel $(P<0.0001)$. Due to technical difficulties during sample preparation, measurements of monocyte infiltration could not be obtained in the nontransgenic strain treated with rosuvastatin. Rosuvastatin treatment significantly decreased the mean number of perivascular monocytes in transgenic SHR expressing human CRP. \#Significant difference compared to the other two groups $(P<0.05$ after adjustment for multiple comparisons). Oxidative tissue damage was assessed by measuring conjugated dienes in heart. Two-way ANOVA showed a significant treatment $\times$ strain interaction effect on conjugated dienes $(P=0.0068) . *$ Denotes that rosuvastatin treatment induced a significant reduction in conjugated diene levels in the CRP-transgenic SHR strain. Rosuvastatin did not affect conjugated diene levels in the nontransgenic SHR strain. CRP, C-reactive protein; SHR, spontaneously hypertensive rat; Rosuv, rosuvastatin; IL6, interleukin 6; and TNF $\alpha$, tumor necrosis factor alpha.

Table 1 Effects of rosuvastatin treatment on metabolic parameters in CRP-transgenic SHR strain and in nontransgenic SHR rat

\begin{tabular}{|c|c|c|c|c|}
\hline & \multicolumn{2}{|c|}{ CRP-Transgenic SHR } & \multicolumn{2}{|c|}{ Nontransgenic SHR } \\
\hline & No treatment & Rosuvastatin & No treatment & Rosuvastatin \\
\hline Body weight $(g)^{\S}$ & $434 \pm 9$ & $421 \pm 7$ & $469 \pm 5$ & $488 \pm 6$ \\
\hline Fasting serum glucose (mmol/L) & $4.1 \pm 0.2$ & $4.1 \pm 0.2$ & $4.6 \pm 0.3$ & $4.0 \pm 0.1$ \\
\hline Nonfasting serum glucose (mmol/L) & $8.6 \pm 0.3$ & $8.3 \pm 0.6$ & $7.4 \pm 0.6$ & $9.0 \pm 0.4$ \\
\hline $\begin{array}{l}\text { AUC for glucose in OGTT } \\
(\mathrm{mmol} / \mathrm{L} / 2 \mathrm{~h})\end{array}$ & $795 \pm 56$ & $811 \pm 10$ & $782 \pm 19$ & $790 \pm 20$ \\
\hline Fasting serum insulin $(\mathrm{nmol} / \mathrm{L})^{\#}$ & $0.08 \pm 0.02$ & $0.04 \pm 0.01$ & $0.05 \pm 0.01$ & $0.03 \pm 0.01$ \\
\hline Nonfasting serum insulin $(\mathrm{nmol} / \mathrm{L})^{\S}$ & $0.57 \pm 0.06$ & $0.34 \pm 0.05^{*}$ & $0.44 \pm 0.06$ & $0.50 \pm 0.05$ \\
\hline Serum insulin during OGTT $(\mathrm{nmol} / \mathrm{L})^{\S}$ & $0.20 \pm 0.03$ & $0.07 \pm 0.01 *$ & $0.08 \pm 0.01$ & $0.06 \pm 0.01$ \\
\hline Serum triglycerides (mmol/L) & $1.45 \pm 0.07$ & $1.12 \pm 0.12$ & $1.35 \pm 0.15$ & $1.47 \pm 0.11$ \\
\hline Serum cholesterol $(\mathrm{mmol} / \mathrm{L})^{\#}$ & $2.29 \pm 0.10$ & $2.67 \pm 0.16$ & $2.19 \pm 0.08$ & $2.40 \pm 0.06$ \\
\hline Liver triglycerides $(\mu \mathrm{mol} / \mathrm{g})^{\S}$ & $9.80 \pm 0.29$ & $6.48 \pm 0.34^{\star}$ & $10.95 \pm 0.78$ & $11.54 \pm 1.19$ \\
\hline Liver TBARs $(\mathrm{nmol} / \mathrm{mg})^{\S}$ & $1.29 \pm 0.07$ & $0.74 \pm 0.06^{*}$ & $0.86 \pm 0.06$ & $0.57 \pm 0.04^{\star}$ \\
\hline
\end{tabular}

Two-way ANOVA results: ${ }^{\S}$ significant $P<0.05$ treatment $\times$ strain interaction-the effects of treatment with rosuvastatin are influenced by the type of strain tested (CRP-transgenic SHR or Nontransgenic SHR). ${ }^{*} P<0.05$ significance for comparisons between treated and untreated animals within a strain by Holm-Sidak testing (i.e., comparisons between a rosuvastatin treated strain and the same strain without treatment). Significant overall strain effects or treatment effects in the two-way ANOVA are flagged only for those measurements where no treatment $\times$ strain interaction was detected. ${ }^{\#} P<0.05$ significance for overall treatment effect in the absence of a treatment $\times$ strain interaction effect. No overall strain effects were detected in the absence of a treatment $\times$ strain interaction effect. 
serum triglyceride levels or cholesterol levels (Table 1). Rosuvastatin treatment actually tended to cause a mild increase in cholesterol levels in both strains. However, significant treatment $\times$ strain interactions were observed on liver triglyceride levels, $P=0.03$ (Table 1). Rosuvastatin decreased hepatic triglycerides in the CRP-transgenic strain but not in the nontransgenic strain (Table 1). In addition, rosuvastatin appeared to decrease liver lipid peroxidation products (TBARS) more in the CRP-transgenic strain than in the nontransgenic strain (Table 1 ).

There was a significant treatment $\times$ strain interaction effect on epididymal fat pad weight $(P=0.039)$ as rosuvastatin treatment was associated with a reduction in epididymal fat in the CRPtransgenic strain but had little or no effect on epididymal fat in the nontransgenic strain (Figure 3). We also observed significant treatment $\times$ strain interaction effects of rosuvastatin on insulinstimulated incorporation of glucose into adipose tissue lipids $(P=0.004)$ and on adrenaline-stimulated lipolysis $(P=0.008)$. Specifically, in the CRP-transgenic strain, but not in the nontransgenic strain, rosuvastatin treatment increased insulin-stimulated incorporation of glucose into adipose tissue lipids and increased adrenaline-stimulated lipolysis (Figure 3).

\section{Effects on Hepatic Gene Expression Profiles}

The overall effects of rosuvastatin on hepatic gene expression levels in the CRP-transgenic strain were similar to those in the nontransgenic strain, and we did not observe any significant treatment $\times$ strain interactions (Table S1 and S2; Data S1). Although we did not detect any significant treatment $\times$ strain interactions, we observed significant rosuvastatin treatment effects on expression levels of 729 transcript clusters and significant strain effects on expression of 46 transcript clusters (Table S1). Table 2 displays genes from the KEGG pathways with the most significant results in the g:Profiler enrichment analysis of the rosuvastatin treatment effects. These pathways included genes related to toll-like receptor signaling (Irak3, Irak4, Myd88, Tlr4), c-Jun N-terminal MAPK signaling (Mapk2k7, Mapk9), extracellular signal-regulated Raf/Mek/Erk signaling (Kras, Map3k1, Mapk2k1), nuclear factor kappa B signaling (Nfkbia), and genes involved in innate immune and antiviral defensive mechanisms (Stat2, Trim25).

\section{Discussion}

The primary finding in the current studies is that rosuvastatin can attenuate inflammation that is dependent on human CRP and can do so, without reducing circulating levels of human CRP. Rosuvastatin also protected against myocardial inflammation and oxidative damage in human CRP-transgenic rats. These observations indicate that rosuvastatin may inhibit inflammation by interfering with the ability of human CRP to activate inflammatory pathways, not just by reducing circulating concentrations of human CRP, or by inhibiting other causes of inflammation.

In previous studies, rosuvastatin was found to protect SpragueDawley rats against increases in blood pressure, aortic vessel oxidative stress, and impaired aortic vascular relaxation induced by postnatal administration of an adeno-associated viral vector containing the cDNA for human CRP [20]. To our knowledge, the
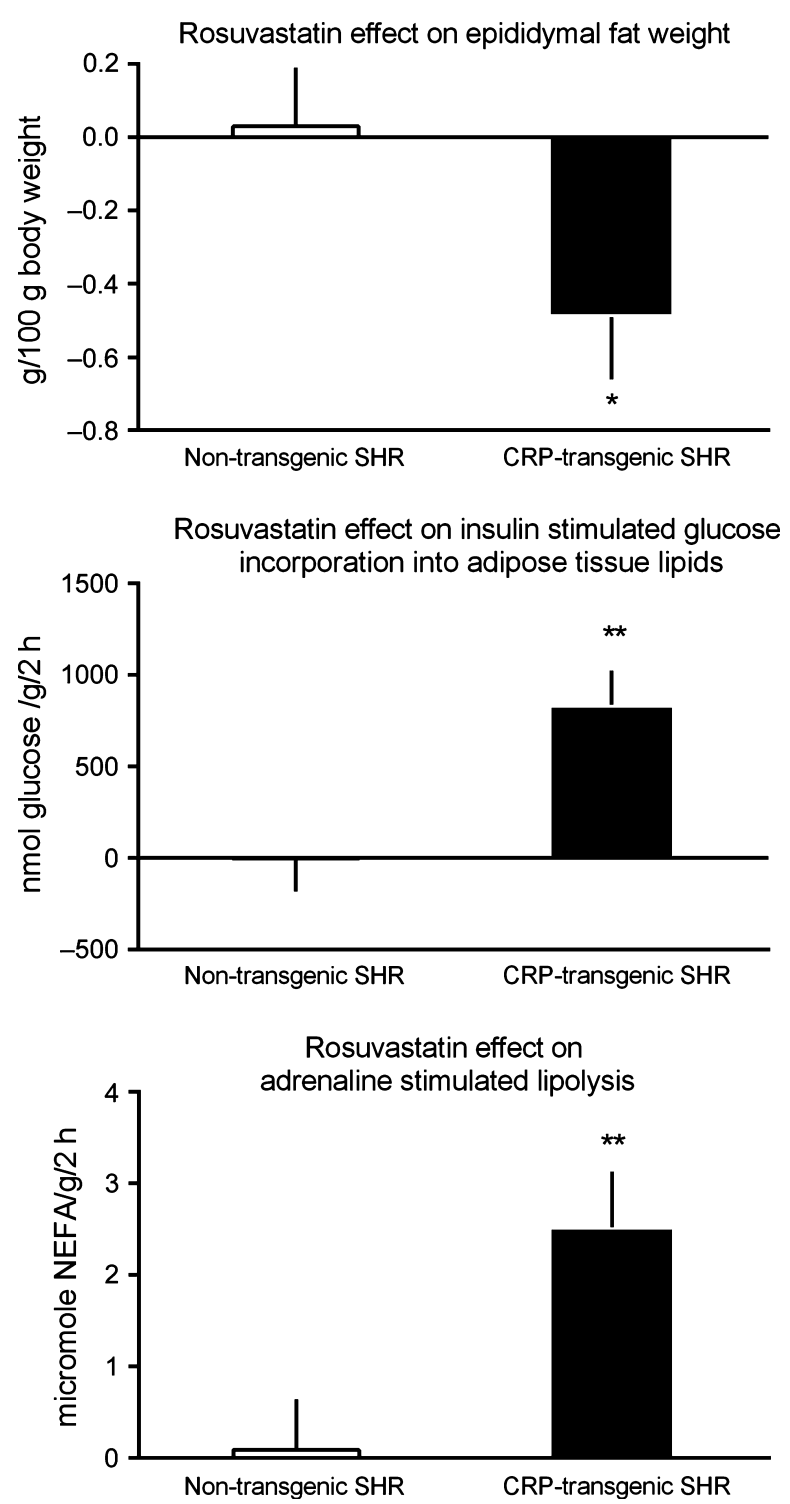

Figure $\mathbf{3}$ Rosuvastatin-induced changes in adipose tissue mass and function dependent on presence of human CRP. Top panel: changes in epididymal fat pad weight induced by rosuvastatin in the CRP-transgenic strain and the nontransgenic strain. Results are adjusted for changes in fat pad weight in untreated animals. Rosuvastatin treatment was associated with a reduction in epididymal fat mass in the CRP-transgenic strain but had little or no effect on epididymal fat mass in the nontransgenic strain. Middle panel: changes in insulin-stimulated incorporation of glucose into adipose tissue lipids induced by rosuvastatin. In the CRP-transgenic strain, but not in the nontransgenic strain, rosuvastatin treatment increased insulin-stimulated incorporation of glucose into adipose tissue lipids. Bottom panel: changes in adrenalinestimulated lipolysis induced by rosuvastatin. In the CRP-transgenic strain, but not in the nontransgenic strain, rosuvastatin treatment increases adrenaline-stimulated lipolysis. $* P<0.05$ for strain $x$ treatment interaction effect in the two-way ANOVA and a significant within strain treatment effect by Holm-Sidak testing. $* * P<0.01$ for strain $x$ treatment interaction effect in the two-way ANOVA and a significant within strain treatment effect by Holm-Sidak testing. 
Table 2 List of genes from top KEGG pathways showing rosuvastatin treatment effects

\begin{tabular}{|c|c|c|}
\hline Pathway & $P$ value & Genes \\
\hline Neurotrophin signaling & $1.91 \mathrm{e}-03$ & 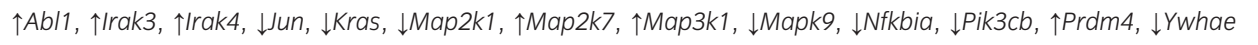 \\
\hline $\begin{array}{l}\text { Aldosterone-regulated } \\
\text { sodium reabsorption }\end{array}$ & $1.89 \mathrm{e}-02$ & $\downarrow$ Atp1b3, $\uparrow$ Kcnj1,$\downarrow$ Kras, $\uparrow N e d d 4 I, \downarrow P i k 3 c b, \uparrow S c n n 1 a, \downarrow S I c 9 a 3 r 2$ \\
\hline Influenza A & $2.97 e-02$ & $\begin{array}{l}\downarrow \text { Hspa1b, } \uparrow \text { Irak4, } \downarrow \text { Jun, } \downarrow \text { Map2k1, } \text { Map2k7, } \downarrow \text { Mapk9, } \text { MMyd88, } \downarrow \text { Nfkbia, } \uparrow \text { Pde11a }, \downarrow \text { Pik3cb, } \downarrow \text { Prss2, } \uparrow \text { Stat2, } \uparrow T \text { TIr4, } \\
\uparrow \text { Trim25 }\end{array}$ \\
\hline
\end{tabular}

$\uparrow$ and $\downarrow$ denote up- and downregulated, respectively, in rosuvastatin treated versus placebo rats; enrichment analysis was performed by g:Profiler with a simulation based analytical threshold for significance estimation.

current studies are the first to show that a statin can protect against systemic and myocardial inflammation induced by endogenously generated human CRP and can do so in the absence of reductions in serum levels of human CRP. In addition, we report novel findings that in the presence of increased levels of human CRP, rosuvastatin can enhance adrenaline-stimulated lipolysis and protect against accumulation of visceral fat. These findings should motivate further studies examining the extent to which the effects of rosuvastatin on adipose tissue mass and function are related to an ability to block pro-inflammatory effects of human CRP in fat. In the current studies, we did not measure the effects of rosuvastatin on blood pressure. Because increased blood pressure can promote oxidative stress and inflammation, it should be recognized that the antioxidant and antiinflammatory effects of rosuvastatin in vivo may also be secondary to the ability of rosuvastatin to limit increases in blood pressure otherwise induced by human CRP.

In the CRP-transgenic rat, serum concentrations of human CRP are about 2-4-fold greater than serum levels of endogenous rat CRP. Thus, relative to the usual serum concentrations of CRP in the rat [21], this transgenic model has only moderately increased serum levels of CRP. While the CRP levels in this transgenic model are not grossly increased for the rat, the concentrations of human CRP achieved are 200-600-fold greater than the median serum level of CRP $(0.8 \mathrm{mg} / \mathrm{L})$ observed in healthy young blood donors [22]. However, in the presence of an acute inflammatory stimulus in humans, serum concentrations of CRP can achieve levels more than 600 times the median normal level of CRP [23]. Moreover, in some patient populations, approximately $25 \%$ of subjects exhibit CRP levels 10 -fold greater than the median level of CRP of healthy blood donors, and many show levels that are 100-500-fold greater [24]. Thus, at least periodically, many people are being exposed to high levels of CRP similar to those attained in the current model. It has been proposed that such increases in CRP could become injurious in the presence of other risk factors for or sources of inflammation and tissue damage [25].

Because rosuvastatin is known to be highly concentrated in liver [19], we also performed some exploratory studies on its ability to affect hepatic levels of triglycerides, lipid peroxidation products, and gene expression. Rosuvastatin reduced hepatic levels of triglycerides and lipoperoxidation products in the CRPtransgenic rats but had comparatively little or no effect on these parameters in the nontransgenic rats. Previous studies have shown that rosuvastatin or pravastatin can also protect against hepatic steatosis in mice fed a high-fat diet, partly through the antiinflammatory effects of these drugs and their ability to prevent mitochondrial damage and preserve efficiency of beta oxidation $[26,27]$. This raises the possibility that in the CRP-transgenic rats, rosuvastatin might also be protecting against hepatic steatosis in part through its ability to prevent human CRP from causing inflammation and mitochondrial dysfunction.

Genome-wide expression analysis revealed significant effects of rosuvastatin treatment on hepatic gene expression levels in both the CRP-transgenic strain and the nontransgenic strain. However, we did not observe any significant treatment $\times$ strain interaction effects on hepatic gene expression levels. Nevertheless, it is possible that gene pathways generally affected by rosuvastatin treatment might still be contributing to some of the protective antiinflammatory effects of rosuvastatin observed in transgenic SHR expressing human CRP. For example, rosuvastatin treatment was observed to affect expression of genes related to toll-like receptor and NF- $\kappa \mathrm{B}$ signaling pathways, both of which are relevant to the pathogenesis of inflammation.

In summary, we have found that rosuvastatin can protect against pro-inflammatory effects of human CRP and can do so in a manner that is not strictly dependent on achieving a reduction in circulating levels of human CRP. These findings raise the possibility that treatments that both reduce CRP levels and interfere with pro-inflammatory properties of CRP may be more effective in reducing inflammation and cardiovascular risk than those that reduce CRP levels alone.

\section{Acknowledgments}

This work was supported by Grant LL1204 (within the ERC CZ program) from the Ministry of Education, Youth and Sports of the Czech Republic to MP; Grant 13-04420S from the Czech Science Foundation to MP; Grant NT/14325 from the Ministry of Health of the Czech Republic to MP; Grant MH CZ - DRO ("Institute for Clinical and Experimental Medicine - IKEM, IN 0002301") to LK; grant from the European Community's Seventh Framework Program (FP7/2007-2013) under agreement HEALTH-F4-2010241504 (EURATRANS) to NH.

\section{Conflict of Interest}

TWK is a recipient of lecture honoraria from the manufacturer of rosuvastatin (Astra Zeneca). 


\section{Author Contributions}

J.S., V.Z., V.L., M.S., P.M., V.S., O.O., and L.K. performed the laboratory-based experiments, M.M. performed the histological experiments. N.H., H.S., and K.S. provided gene expression data and analysis. M.P., L.K., and T.W.K. planned the experiments. M.P. and T.W.K. wrote the manuscript with input and discussion from all of the co-authors.

\section{References}

1. Ridker PM, Kastelein JJ, Genest J, Koenig W. C-reactive protein and cholesterol are equally strong predictors of cardiovascular risk and both are important for quality clinical care. Eur Heart J 2013;34:1258-1261.

2. Koenig W. High-sensitivity C-reactive protein and atherosclerotic disease: from improved risk prediction to risk-guided therapy. Int J Cardiol 2013;168:5128-5134.

3. Scirica BM, Morrow DA. Is C-reactive protein an innocent bystander or proatherogenic culprit? The verdict is still out Circulation 2006; 113:2128-2134.

4. Verma S, Devaraj S, Jialal I. Is C-reactive protein an innocent bystander or proatherogenic culprit? C-reactive protein promotes atherothrombosis Circulation 2006; 113:2135-2150

5. Paraskevas KI, Mikhailidis DP. C-reactive protein (CRP): more than just an innocent bystander? Curr Med Res Opin 2008;24:75-78.

6. Yousuf O, Mohanty BD, Martin SS, et al. High-sensitivity C-reactive protein and cardiovascular disease: a resolute belief or an elusive link? J Am Coll Cardiol 2013;62: 397-408.

7. Bisoendial RJ, Boekholdt SM, Vergeer M, Stroes ES, Kastelein JJ. C-reactive protein is a mediator of cardiovascular disease. Eur Heart J 2010;31:2087-2091.

8. Oh J, Teoh H, Leiter LA. Should C-reactive protein be a target of therapy? Diabetes Care 2011;34(Suppl 2):S155-S160.

9. Elliott P, Chambers JC, Zhang W, et al. Genetic Loci associated with C-reactive protein levels and risk of coronary heart disease. JAMA 2009; 302:37-48.

10. Wensley F, Gao P, Burgess S, et al. Association between $\mathrm{C}$ reactive protein and coronary heart disease: mendelian randomisation analysis based on individual participant data. $B M J$ 2011;342: d548.

11. Burgess S, Butterworth A, Malarstig A, Thompson SG. Use of Mendelian randomisation to assess potential benefit of clinical intervention. BMJ 2012;345:e7325.

12. Kumaresan PR, Devaraj S, Huang W, Lau EY, Liu R, Lam KS, Jialal I. Synthesis and characterization of a novel inhibitor of C-reactive protein-mediated proinflammatory effects. Metab Syndr Relat Disord 2013;11:177184.

13. Ridker PM, Danielson E, Fonseca FA, et al. Rosuvastatin to prevent vascular events in men and women with elevated C-reactive protein. N Engl J Med 2008;359:2195-2207.

14. Devaraj S, Singh U, Jialal I. The evolving role of C-reactive protein in atherothrombosis. Clin Chem 2009;55:229-238.

15. Pepys MB, Hirschfield GM, Tennent GA, et al. Targeting C-reactive protein for the treatment of cardiovascular disease. Nature 2006;440: 1217-1221.

16. Pravenec M, Kajiya T, Zidek V, et al. Effects of human C-reactive protein on pathogenesis of features of the metabolic syndrome. Hypertension 2011;57:731-737.

17. Koike T, Kitajima S, Yu Y, et al. Human C-reactive protein does not promote atherosclerosis in transgenic rabbits. Circulation 2009;120:2088-2094.

18. Malinska H, Oliyarnyk O, Hubova M, et al. Increased liver oxidative stress and altered PUFA metabolism precede development of non-alcoholic steatohepatitis in SREBP-la transgenic spontaneously hypertensive rats with genetic predisposition to hepatic steatosis. $\mathrm{Mol}$ Cell Biochem 2010;335:119-125.

19. Nezasa K, Takao A, Kimura K, Takaichi M, Inazawa K, Koike M. Pharmacokinetics and disposition of rosuvastatin, a new 3-hydroxy-3-methylglutaryl coenzyme A reductase inhibitor, in rat. Xenobiotica 2002;32:715-727.

20. Li X, Yang G, Zhao G, et al. Rosuvastatin attenuates the elevation in blood pressure induced by overexpression of human C-reactive protein. Hypertens Res 2011;34:869-875.

21. de Beer FC, Baltz ML, Munn EA, et al. Isolation and characterization of $\mathrm{C}$-reactive protein and serum amyloid $\mathrm{P}$ component in the rat. Immunology 1982;45:55-70.

22. Shine B, de Beer FC, Pepys MB. Solid phase radioimmunoassays for human $\mathrm{C}$-reactive protein. Clin Chim Acta 1981;117:13-23.

23. Pepys MB, Hirschfield GM. C-reactive protein: a critical update. J Clin Invest 2003;11 1:18051812 .

24. Johnsson H, Panarelli M, Cameron A, Sattar N. Analysis and modelling of cholesterol and high-density lipoprotein cholesterol changes across the range of $\mathrm{C}$-reactive protein levels in clinical practice as an aid to better understanding of inflammation-lipid interactions. Ann Rheum Dis 2013; [Epub ahead of print].

25. Casas JP, Shah T, Hingorani AD, Danesh J, Pepys MB. C-reactive protein and coronary heart disease: a critical review. J Intern Med 2008;264:295-314.

26. Neto-Ferreira R, Rocha VN, Souza-Mello V, Mandarim-de-Lacerda CA, de Carvalho JJ. Pleiotropic effects of rosuvastatin on the glucose metabolism and the subcutaneous and visceral adipose tissue behavior in $\mathrm{C} 57 \mathrm{Bl} / 6$ mice. Diabetol Metab Syndr 2013;5:32.

27. Araki K, Masaki T, Katsuragi I, Kakuma T, Yoshimatsu H. Effects of pravastatin on obesity, diabetes, and adiponectin in diet-induced obese mice. Obesity (Silver Spring) 2008;16: 2068-2073.

\section{Supporting Information}

Additional Supporting Information may be found in the online version of this article:

Data S1. Supplemental methods and results.
Table S1. Summary of hepatic gene expression profiling results.

Table S2. Data and count. 